\title{
Aspergillus fumigatus in the cystic fibrosis lung: pros and cons of azole therapy
}

Pierre-Régis Burgel ${ }^{1,2}$

André Paugam ${ }^{2,3}$

Dominique Hubert ${ }^{1,2}$

Clémence Martin ${ }^{1,2}$

'Department of Respiratory Medicine, Cochin Hospital, Assistance Publique - Hôpitaux de Paris, ${ }^{2}$ Université Paris Descartes, Sorbonne Paris Cité, ${ }^{3}$ Parasitology-Mycology Laboratory, Cochin Hospital, Assistance Publique - Hôpitaux de Paris, Paris, France
This article was published in the following Dove Press journal:

Infection and Drug Resistance

20 September 2016

Number of times this article has been viewed

\begin{abstract}
Aspergillus fumigatus is the main fungus cultured in the airways of patients with cystic fibrosis (CF). Allergic bronchopulmonary aspergillosis occurs in $\sim 10 \%$ of CF patients and is clearly associated with airway damage and lung function decline. The effects of A. fumigatus colonization in the absence of allergic bronchopulmonary aspergillosis are less well established. Retrospective clinical studies found associations of $A$. fumigatus-positive cultures with computed tomography scan abnormalities, greater risk of CF exacerbations and hospitalizations, and/or lung function decline. These findings were somewhat variable among studies and provided only circumstantial evidence for a role of $A$. fumigatus colonization in CF lung disease progression. The availability of a growing number of oral antifungal triazole drugs, together with the results of nonrandomized case series suggesting positive effects of azole therapies, makes it tempting to treat $\mathrm{CF}$ patients with these antifungal drugs. However, the only randomized controlled trial that has used itraconazole in CF patients showed no significant benefit. Because triazoles may have significant adverse effects and drug interactions, and because their prolonged use has been associated with the emergence of azole-resistant $A$. fumigatus isolates, it remains unclear whether or not CF patients benefit from azole therapy.
\end{abstract}

Keywords: itraconazole, voriconazole, posaconazole, azole resistance, allergic bronchopulmonary aspergillosis

\section{Introduction}

Cystic fibrosis (CF) is an autosomal recessive genetic disease characterized by mutations in the gene encoding for the CFTR protein, which regulates transport of chloride and bicarbonate ions at the epithelium surface. The disease, which was lethal in the first years of life 50 years ago, has benefited from several therapeutic advances, resulting in a major increase in life expectancy with most patients reaching adult age and median survival estimated at least to be 40 years in developed countries. ${ }^{1}$ As a result, the number of adult CF patients already outgrows the number of CF children in many countries, ${ }^{2}$ and demographic forecasts predict a marked increase in the number of CF (mostly adult) patients in the next decade. ${ }^{3}$

\section{Fungi in the lung in CF patients - $a$ focus on A. fumigatus}

The CF lung is highly vulnerable to respiratory infections that promote airway inflammation and remodeling. ${ }^{4,5}$ Most available data on infection in CF airways are based on sputum bacteriological cultures that identified Gram-negative (eg, Pseudomonas
Correspondence: Pierre-Régis Burgel Service de Pneumologie, Hôpital Cochin27 Rue du Faubourg Saint Jacques, 75014 Paris, France

Tel +33 I 584| 2367

Fax +33 | 46338253

Email pierre-regis.burgel@aphp.fr 
aeruginosa) and Gram-positive (eg, Staphylococcus aureus) bacteria as predominant pathogens. ${ }^{6}$ More recently, it has become clear that other infectious agents (eg, nontuberculous mycobacteria and fungi) may contribute to chronic lung disease in CF patients. ${ }^{7}$ Although several fungi can be cultured in CF airway secretions, Aspergillus is the main fungus cultured in CF airways. ${ }^{8,9}$ Recent studies that used mass spectrometry and/or molecular methods confirmed that among Aspergillus spp. (ie, Aspergillus fumigatus, Aspergillus terreus, and Aspergillus niger), A. fumigatus is by far the most common species isolated in the sputum of CF patients. ${ }^{9,10}$

Registry data suggest that $A$. fumigatus is cultured in $10 \%-25 \%$ of CF patients, ${ }^{11}$ and is reported more frequently in adolescents and adults. ${ }^{12}$ However, these data likely underestimate the true prevalence of $A$. fumigatus-positive culture in $\mathrm{CF}$ airway fluids because young children (who usually produce less sputum) are less likely to be sampled and because sampling practices in older children and adults may vary among different CF centers. Studies that have used systematic sputum sampling in adult CF patients have usually reported higher, although variable, rates of $A$. fumigatuspositive cultures. For example, a recent study in 146 adult CF patients reported positive sputum cultures for $A$. fumigatus in $27 \%$ of patients, ${ }^{13}$ and studies at our center reported positive sputum cultures for $A$. fumigatus in up to $57 \%$ of adult patients. ${ }^{8,14}$ Variations in the percentage of patients colonized with $A$. fumigatus could be explained by various factors including environmental exposure, interactions with other $\mathrm{CF}$ pathogens, and therapeutic interventions. Rocchi et al found marked differences in A. fumigatus conidia concentrations in the home of $16 \mathrm{CF}$ patients. ${ }^{15}$ Although the latter study was not designed to address the relationship between $A$. fumigatus exposure load and positive culture in sputum, it seems likely that exposure to higher load of $A$. fumigatus could result in higher rates of $A$. fumigatus colonization. Delhaes et al reported that $P$. aeruginosa is less likely to be associated with $A$. fumigatus than Candida albicans, ${ }^{16}$ and Briard et al found that $P$. aeruginosa products decrease $A$. fumigatus growth in vitro. ${ }^{17}$ Further, steroids are well known to promote A. fumigatus growth, and Noni et al reported increased risk of A. fumigatus-positive cultures in CF subjects treated with inhaled steroids. ${ }^{18}$ The chronic use of inhaled antibiotics (eg, tobramycin or colomycin) has also been associated with increased risk of $A$. fumigatus sputum-positive cultures, ${ }^{12,19}$ whereas a recent study reported that a single course of intravenous antibiotics targeting $P$. aeruginosa reduced the presence of Aspergillus (as detected by polymerase chain reaction [PCR] and galactomannan; explained in the "Methods for detecting $A$. fumigatus or immune response to $A$. fumigatus in CF patients" section) in adult CF sputum. ${ }^{20}$ However, in the latter study, only two of 26 patients had positive sputum cultures for Aspergillus. ${ }^{20}$ Long-term use of azithromycin was also associated with increase in A. fumigatus-positive sputum cultures. ${ }^{21}$

\section{Why does Aspergillus stay in CF lungs?}

Mechanisms leading to frequent $A$. fumigatus presence in $\mathrm{CF}$ airways are not fully understood. A. fumigatus is present in the environment and regularly enters the airways with inhaled air. The repeated presence of $A$. fumigatus isolates in the CF lungs may result either from chronic colonization with a single (or a predominant) isolate or from recurrent colonization by genotypes that succeed each other. ${ }^{22}$ Host defenses against inhaled $A$. fumigatus involve both innate immune responses (eg, mucociliary clearance, airway epithelial cells, and phagocytes such as neutrophils and macrophages) and adaptive immune responses via $\mathrm{T}$ cell-mediated responses. ${ }^{23,24}$ An autopsy study identified $A$. fumigatus within mucous plugs in $\mathrm{CF}$ airways, ${ }^{25}$ suggesting a role for defective mucociliary clearance in $A$. fumigatus persistence in $\mathrm{CF}$ airways. A direct role for CFTR in the clearance of inhaled $A$. fumigatus spores by airway epithelial cells has also been suggested. ${ }^{26}$ Further, CFTR-deficient T-lymphocytes response to A. fumigatus challenge is biased toward Th-2-dependent immune response involving $\operatorname{IgE} .{ }^{27} \mathrm{~A}$ recent study suggested that the selective proteolysis of the soluble pattern recognition receptor pentraxin 3 (PTX3 may contribute to the persistence of A. fumigatus in the lung of CF patients). ${ }^{28}$ Regardless of the mechanisms, the role of CFTR defect in A. fumigatus colonization in $\mathrm{CF}$ airways has been confirmed by recent human studies with drugs directly targeting the CFTR dysfunction. Ivacaftor, the first marketed CFTR potentiator, reduced the occurrence of $A$. fumigatus-positive sputum cultures in $\mathrm{CF}$ patients with a G551D mutation. ${ }^{29}$

\section{Methods for detecting A. fumigatus or immune response to $A$. fumigatus in CF patients}

The most classical method for detecting A. fumigatus and other fungi in CF airways is the culture of airway secretion (eg, sputum, bronchoalveolar lavage fluid) on appropriate culture medium (eg, Sabouraud). Investigators have highlighted the technical differences (eg, use of different culture medium, use of different lengths of time for cultures) among 
laboratories processing CF samples. ${ }^{30}$ Use of specific medium or long incubation time may allow for recovering other fungi (eg, Scedosporium sp. and Exophiala dermatitidis) ${ }^{31}$ but is unlikely to affect the rates of positive culture for A. fumigatus. However, culture-based identification of $A$. fumigatus is not sensitive, and investigators have developed cultureindependent methods for assessing A. fumigatus in $\mathrm{CF}$ samples. These techniques include detecting $A$. fumigatus nucleic acids or A. fumigatus products (eg, galactomannan).

Baxter et al compared real-time PCR targeting a portion of the 18S rDNA of Aspergillus with fungal cultures in homogenized and sonicated CF sputum. ${ }^{32}$ The authors tested 121 sputum samples obtained from $\mathrm{CF}$ patients and found that $33(30 \%)$ were culture and PCR positive, 48 (43\%) samples were culture negative, but PCR positive $(P<0.0001)$, and 30 (27\%) samples were culture and PCR negative. ${ }^{32}$ Comparable results have been obtained by another group, ${ }^{33}$ suggesting that PCR is more sensitive than culture for detecting A. fumigatus in $\mathrm{CF}$ sputum. In the near future, high-throughput methods targeting fungal ribosomal RNA may be used to examine fungal diversity in $\mathrm{CF}$ airways, ${ }^{16,34}$ but these techniques have not yet reached routine use.

Galactomannan is produced during the logarithmic growth phase of the fungus; thus, its presence is considered to represent active growth of the fungus, associated with infection rather than colonization. Although galactomannan is most likely related to A. fumigatus in CF sputum, galactomannan is also a component of other fungal cell walls and could be present in the absence of $A$. fumigatus. ${ }^{35,36}$ Serum galactomannan is often used to detect invasive aspergillosis in immunocompromised patients, but invasive pulmonary aspergillosis is a rare finding in CF patients. ${ }^{37}$ Warren et al tested sera from 138 pediatric and adult CF patients for the presence of galactomannan: all serum samples were negative for galactomannan, regardless of the presence of positive cultures for $A$. fumigatus in sputum. ${ }^{38}$ These data suggest that testing for serum galactomannan in stable CF patients is of limited interest. More recently, Baxter et al reported that galactomannan could be assessed with good reproducibility in homogenized sputum. ${ }^{13}$ The authors suggested that negative sputum galactomannan could be useful for the identification of CF patients without significant $A$. fumigatusrelated disease.

Immune responses to $A$. fumigatus are complex events, ${ }^{36}$ which may involve, among other mechanisms, IgG and/or IgE production. The detection of IgG against $A$. fumigatus can be performed using enzyme-linked immunosorbent assays, but immunoprecipitin detection is usually considered the reference technique. ${ }^{39,40}$ This technique suffers from the lack of standardization, and results may vary across different laboratories. More recently, a Western blot for detecting IgG has been commercialized and may contribute to more standardized detection of IgG against A. fumigatus. ${ }^{41}$ However, usefulness of this assay in CF patients has not yet been established. The detection of IgE-mediated immune response to A. fumigatus may involve A. fumigatus skin prick test and/ or the detection of total $\mathrm{IgE}$ and $\operatorname{IgE}$ that recognizes various A. fumigatus antigens in serum. ${ }^{42}$

\section{Clinical consequences of A. fumigatus in CF patients}

Only rare cases of invasive pulmonary aspergillosis or of aspergilloma have been reported in CF patients, and these consequences of $A$. fumigatus will not be further discussed. By contrast, IgE sensitization defined as positive skin test to A. fumigatus and/or high $A$. fumigatus-specific serum IgE concentration is present in up to $40 \%$ of CF patients. ${ }^{43}$ Allergic bronchopulmonary aspergillosis (ABPA) occurs in $\sim 10 \%$ of CF patients. ${ }^{44}$ Although no universally accepted definition of ABPA exists, the disease is usually characterized by $A$. fumigatus sensitization, $A$. fumigatus $\mathrm{IgG}$ response with clinical symptoms (eg, wheezing), and computed tomography (CT) scan manifestations. ${ }^{44}$ Both IgE sensitization and ABPA have been consistently associated with clinically meaningful outcomes (eg, lung function decline and/or pulmonary exacerbations) in children and adults with CF. Wojnarowski et al reported that $26 \%$ of a cohort of $118 \mathrm{CF}$ children were sensitized to A. fumigatus (based on the results of total IgE, specific $A$. fumigatus IgE, and skin prick tests). ${ }^{45}$ The authors reported lower lung function in CF children with positive A. fumigatus skin prick test and/or specific IgE and elevated total IgE. ${ }^{45}$ In a retrospective study, Kraemer et al analyzed serial lung function tests in $122 \mathrm{CF}$ children. ${ }^{46}$ The authors reported that $A$. fumigatus sensitization (ie, increased specific IgE to recombinant $A$. fumigatus antigens) was associated with increased rates of lung function decline in subjects with ABPA and in subjects without ABPA. ${ }^{46}$ The authors suggested that IgE sensitization to A. fumigatus could represent earlyonset ABPA.${ }^{46}$ These data were largely confirmed by reports on mixed populations of children and adults with $\mathrm{CF}^{47,48}$ and in a study of CF adults. ${ }^{49}$ Additionally, Baxter et al found that adult CF patients with $A$. fumigatus sensitization $(\mathrm{n}=33)$ received approximately twice as many days of intravenous antibiotics than those without sensitization $(n=22)$ over a 2-year prospective follow-up study, ${ }^{49}$ a finding that was confirmed in a study by Peetermans et al. ${ }^{48}$ 
Many CF patients without IgE sensitization or ABPA have repeated positive sputum samples when examined for A. fumigatus using cultures or PCR, and these patients are considered to exhibit $A$. fumigatus colonization or infection. ${ }^{50}$ Several studies have assessed the association of A. fumigatus colonization with clinically relevant outcomes in CF patients, and these studies have reported conflicting results. Ramsey et al performed bronchoalveolar lavage at age 3 months, 1 year, and 2 years in CF children diagnosed by newborn screening and correlated their findings with lung function (as measured by forced expiratory volume in the first three-quarters of a second, $\mathrm{FEV}_{0.75}$ ) at early school age (4-8 years).$^{51}$ Early-life respiratory tract infections with proinflammatory pathogens including Haemophilus influenzae, Streptococcus pneumoniae, and Aspergillus sp. were associated with subsequent reductions in lung function (measured by $\mathrm{FEV}_{0.75}$ ). ${ }^{51}$ Fillaux et al reported larger decline in forced expiratory volume in 1 second $\left(\mathrm{FEV}_{1}\right)$ in CF patients with persistent $A$. fumigatus carriage (defined by either a persistent $A$. fumigatus-positive culture or positive Aspergillus IgG precipitins of A. fumigatus compared with the control group) ${ }^{47}$ Amin et al performed a retrospective cohort study in 230 children and adolescents with CF between 1999 and 2006 and reported that $16 \%$ of these patients had persistent ( $\geq 2 /$ year) positive sputum culture for $A$. fumigatus. ${ }^{50}$ Patients with persistent $A$. fumigatus-positive cultures had lower $\mathrm{FEV}_{1}$ (79.2\% vs $86.1 \%$ predicted in the A. fumigatusnegative group) at baseline, and their $\mathrm{FEV}_{1}$ remained $3.61 \%$ predicted lower during the study period. However, it remained unclear whether these findings were independently related to A. fumigatus. ${ }^{50}$ In the latter study, persistence of $A$. fumigatus in sputum was an independent risk factor (relative risk, 1.94) for pulmonary exacerbations requiring hospital admission. ${ }^{50}$ de Boer et al also found that adult CF patients with frequent $\mathrm{CF}$ exacerbations had increased rates of repeated positive cultures for A. fumigatus in sputum, ${ }^{52}$ although it remains unclear whether this association was independent of other risk factors for exacerbations. Overall, these studies are consistent with the suggested proinflammatory role of A. fumigatus on the airway epithelium: Reihill et al reported that incubation of cultured CF airway epithelial cells with A. fumigatus, but not with $C$. albicans, resulted in the production of the proinflammatory cytokines IL-6 and IL- $8 .{ }^{53}$ However, not all studies reported an association between $A$. fumigatus-positive cultures and lung function decline. In a cross-sectional analysis of 259 children and adults with CF, de Vrankrijker et al found that subjects with repeated positive sputum cultures for A. fumigatus had lower lung function and higher rates of hospitalization. ${ }^{12}$ However, these associations were no longer significant in multivariate analysis. ${ }^{12}$ Most importantly, the authors performed a longitudinal analysis of lung function decline in 163 patients followed for 5 years and reported that the duration of $A$. fumigatus colonization did not affect $\mathrm{FEV}_{1}$ decline. ${ }^{12}$ Baxter et al compared $\mathrm{FEV}_{1}$ decline over 2 years in $33 \mathrm{CF}$ patients with repeated positive detection of A. fumigatus in sputum by PCR with $22 \mathrm{CF}$ patients without repeatedly positive PCR. ${ }^{49}$ The authors found no difference in $\mathrm{FEV}_{1}$ decline between the two groups. ${ }^{49}$ Finally, McMahon et al found no difference in $\mathrm{FEV}_{1} \%$ predicted when they cross-sectionally compared $16 \mathrm{CF}$ patients with repeated A. fumigatus-positive sputum cultures with $16 \mathrm{CF}$ patients without A. fumigatus. ${ }^{54}$ The authors further reported greater CT scan abnormalities in A. fumigatus-positive patients and suggested that $\mathrm{FEV}_{1}$ was not sensitive enough to detect changes associated with $A$. fumigatus colonization. ${ }^{54}$ It should be noted that an important limitation of all published studies is that they were designed to examine possible statistical associations between $A$. fumigatus colonization and clinical outcomes. Such data may provide circumstantial evidence but cannot provide causal relationship. We conclude that the relationship between A. fumigatus colonization, lung function decline, and/or CF exacerbation remains unclear and that definitive data on the role of $A$. fumigatus in the clinical course of disease in CF patients will require performing randomized controlled trials using oral (eg, azoles) or inhaled (eg, amphotericin B) antifungal agents. These studies should be designed to examine both microbiological end points (eg, fungus eradication or reduction in fungal load) and clinically meaningful end points (eg, lung function, exacerbations, quality of life, and imaging).

\section{A role for azole therapy in CF patients?}

The availability of oral antifungal drugs (itraconazole, voriconazole, and posaconazole) active against $A$. fumigatus has provided an opportunity to examine the role of $A$. fumigatus in CF lung disease. Initial reports focused on the possible role of azole therapy in CF patients with ABPA. Hilliard et al reported the results of an uncontrolled, open-label, retrospective review of CF patients treated with voriconazole. ${ }^{43}$ These authors reported an improvement in lung function and in A. fumigatus serology in two children with ABPA treated with voriconazole monotherapy, ${ }^{43}$ which was also described in eleven CF children with ABPA treated in combination with immunomodulatory agents. Although these data suggested possible effect of voriconazole in CF patients with ABPA, 
no randomized controlled trial has evaluated the use of antifungal therapies for the treatment of ABPA in CF patients. ${ }^{55}$

In recent years, several uncontrolled open-label case studies reported on the effects of azoles in patients with A. fumigatus colonization in the absence of ABPA. Hilliard et al reported that treatment with voriconazole had no effect on $\mathrm{FEV}_{1}$ in eight $\mathrm{CF}$ children who had recurrent $A$. fumigatus isolates and increased symptoms..$^{43}$ Shoseyov et al used itraconazole or voriconazole in six patients who had A. fumigatus growing in sputum cultures, deteriorating respiratory function, and were not responding to antibacterial therapy. ${ }^{56}$ These authors reported improvement in lung function, nutritional status, and serum IgE concentrations in most patients. ${ }^{56}$ Coughlan et al studied $13 \mathrm{CF}$ patients who were colonized with A. fumigatus and received $400 \mathrm{mg} /$ day of itraconazole for 6 weeks. The authors reported reduced A. fumigatus load in airway secretions, which was associated with improvement in respiratory symptoms and decrease in CT scan abnormalities. ${ }^{33}$ No significant change in $\mathrm{FEV}_{1}$ was observed. ${ }^{33}$ Aaron et al performed the only randomized control study with itraconazole in CF patients with A. fumigatus colonization. ${ }^{57}$ CF subjects with chronic A. fumigatus colonization were randomized to receive itraconazole $(5 \mathrm{mg} / \mathrm{kg} /$ day $)(\mathrm{n}=18)$ or placebo $(\mathrm{n}=17)$ and followed for 24 weeks. Although the study was terminated prematurely due to difficulties in patient recruitment, no difference was observed either in the rate of $\mathrm{CF}$ exacerbations (primary outcome) or in $\mathrm{FEV}_{1}$ decline (secondary outcome). An important limitation of this study is that therapeutic itraconazole blood levels were not achieved in $43 \%$ of patients randomized to itraconazole, underscoring the absolute requirement for therapeutic drug monitoring when using azole therapy (described in the "Adverse effects of azoles" section). In conclusion, the evidence favoring the use of azoles in CF patients with A. fumigatus colonization or with ABPA is exclusively based on case series, and no definitive conclusion on their possible efficacy can be derived from these reports.

\section{Adverse effects of azoles}

An important aspect when examining the harm/benefit ratio of azole therapy in CF patients relates to the safety profile of these drugs. Although azole therapies are usually described as being well tolerated, several adverse effects and drug-drug interactions may occur especially during prolonged treatments. ${ }^{58-60} \mathrm{~A}$ summary of the main adverse effects of commercially available triazoles (ie, itraconazole, voriconazole, and posaconazole) in CF patients is presented in Table 1.

The pharmacokinetics of azole is highly variable among patients and over time in the same patient, underscoring the need for regular therapeutic drug monitoring. ${ }^{61}$ The inability to reach therapeutic concentrations could be related to drug-drug interactions. For example, the absorption of oral itraconazole and posaconazole (but not voriconazole) is promoted by acidic $\mathrm{pH}$ and is thus decreased by coadministration of proton pump inhibitors ${ }^{58,62}$ that are frequently used for the treatment of gastroesophageal reflux in CF patients. The use of rifampicin, for the eradication of methicillin-resistant $S$. aureus or nontuberculous mycobacteria, accelerates itraconazole metabolism.

Azoles exert their effects on A. fumigatus via the inhibition of cytochrome P450 enzymes, which are important for the biosynthesis of sterols that are vital components of fungal cell membranes. ${ }^{58} \mathrm{~A}$ major drug interaction of azoles exists with systemic and inhaled corticosteroids. Although the interaction with prednisolone is considered minor, itraconazole increases serum concentrations of methylprednisolone and dexamethasone. Most importantly, itraconazole also increases serum concentration of the inhaled corticosteroids budesonide and fluticasone. ${ }^{58}$ These effects could result in clinically significant Cushing syndrome with adrenal suppression, ${ }^{63}$ sometimes leading to adrenal insufficiency with fatigue and/or growth failure ${ }^{64} \mathrm{~A}$ recent study that performed systematic synacthen testing in $12 \mathrm{CF}$ patients receiving itraconazole and inhaled fluticasone found that all patients had abnormal synacthen results. ${ }^{65} \mathrm{~A}$ recent

Table I Summary of main adverse effects of azoles in cystic fibrosis patients

\begin{tabular}{llll}
\hline Affected organs & Itraconazole & Voriconazole & Posaconazole \\
\hline $\begin{array}{l}\text { Gastrointestinal } \\
\text { Liver }\end{array}$ & Nausea, vomiting & Nausea, vomiting & Nausea, vomiting \\
Skin & Elevation of liver enzymes & Elevation of liver enzymes & Elevation of liver enzymes \\
& None & Skin rash & None \\
Cardiovascular & & Photosensitivity & \\
Endocrine & Qkin cancers & QT/QTc prolongation \\
& Frequent Cushing syndrome/ & QT/QTc prolongation & Cushing syndrome/ \\
\hline
\end{tabular}

Note: aWhen associated with systemic and/or inhaled steroids. 
report also suggested that similar interactions may occur with voriconazole ${ }^{66}$ or posaconazole. ${ }^{67}$ These findings are of particular relevance because $\sim 40 \%$ of CF patients are treated with inhaled corticosteroids. ${ }^{68}$

Voriconazole, unlike other azoles, is associated with a significant risk of skin rash and photosensitivity. In a retrospective report, Rondeau et al found that $58 \%$ of $31 \mathrm{CF}$ patients treated with voriconazole exhibited photosensitivity despite the use of recommended skin protection. ${ }^{69}$ The authors suggested that for unknown reasons, CF patients appeared more photosensitive to voriconazole than non-CF patients. ${ }^{69}$ These findings are of particular concerns in view of the emerging description of increased risk of skin cancers associated with prolonged voriconazole treatment. ${ }^{70}$

Finally, the use of azoles could be associated with the emergence of azole-resistant $A$. fumigatus isolates.

\section{Emergence of azole resistance in A. fumigatus isolates from CF patients} During the past 15 years, azole resistance has emerged in A. fumigatus isolates ${ }^{71}$ and has been associated with treatment failure in subjects with aspergillosis. ${ }^{72-74}$ This increase in azole-resistant $A$. fumigatus isolates was suggested to occur as a consequence of prolonged therapeutic exposure to azoles in patients treated for chronic cavitary aspergillosis ${ }^{72}$ or as a consequence of environmental exposure to fungicides, which are used in agriculture and have a molecular structure very similar to that of the medical triazoles. ${ }^{75}$ Several methods for assessing resistance of $A$. fumigatus to triazoles have been developed, including culture-based methods and cultureindependent methods that usually detect CYP51 mutations. ${ }^{76}$

Until recently, the prevalence of azole resistance has remained unknown because most clinical laboratories do not routinely perform susceptibility testing of clinical $A$. fumigatus isolates in CF patients. In the past 5 years, several studies from various European countries have systematically examined the prevalence of azole-resistant isolates in CF sputum (Table 2). Although there was some variability among studies, most studies reported that $\sim 5 \%-8 \%$ of $A$. fumigatus isolates obtained in $\mathrm{CF}$ patients were resistant to azoles. In most isolates, mutations in the CYP51A gene were observed. The most prevalent mutation was TR34/L98H, previously reported in azole-naïve patients, and ascribed to exposure of $A$. fumigatus to fungicides used in agriculture. Because many studies were performed in A. fumigatus isolates stored in the microbiology laboratories and contained only limited amount of clinical data, few data are available on the relationship between previous therapeutic exposure to azole and the prevalence of azole-resistant

Table 2 Summary of studies that examined azole resistance in respiratory samples from cystic fibrosis patients

\begin{tabular}{|c|c|c|c|c|c|}
\hline Reference & $\begin{array}{l}\text { Country and study } \\
\text { type }\end{array}$ & Patients, n & $\begin{array}{l}\text { Technique for assessing } \\
\text { azole resistance }\end{array}$ & Azole-resistant strains & CYP5 IA mutations \\
\hline Amorim et al ${ }^{82}$ & $\begin{array}{l}\text { Portugal } \\
\text { Single center (Porto) }\end{array}$ & 11 & CLSI & $0 / 159$ isolates $^{a}$ & $\mathrm{~N} / \mathrm{A}$ \\
\hline Bader et $\mathrm{a}^{83}$ & $\begin{array}{l}\text { Germany } \\
\text { Multicenter }\end{array}$ & $\mathrm{N} / \mathrm{A}$ & $\begin{array}{l}\text { Etest }^{\circledR} \\
\text { EUCAST } \\
\text { CYP5IA sequencing }\end{array}$ & $9 / 163(5.2 \%)$ isolates & $\begin{array}{l}\text { TR34/L98H }(n=3) \\
\text { M220I } \\
\text { F2I9C }\end{array}$ \\
\hline Burgel et al ${ }^{14}$ & $\begin{array}{l}\text { France } \\
\text { Single center (Paris) }\end{array}$ & $|3|$ & $\begin{array}{l}\text { Etest } \\
\text { CLSI } \\
\text { CYP5IA PCR }\end{array}$ & $\begin{array}{l}6 /|3|(4.6 \%) \text { patients } \\
5 / 25(20 \%) \text { patients exposed } \\
\text { to itraconazole within the } \\
\text { previous } 3 \text { years }\end{array}$ & $\begin{array}{l}\text { TR34/L98H }(n=2) \\
\text { M220I } \\
\text { M220R } \\
\text { G54E }\end{array}$ \\
\hline Fischer et al ${ }^{9}$ & $\begin{array}{l}\text { Germany } \\
\text { Single center (Cologne) }\end{array}$ & 221 & $\begin{array}{l}\text { EUCAST } \\
\text { CYP5IA sequencing }\end{array}$ & $6 / 573(1.0 \%)$ isolates & $\begin{array}{l}\text { TR34/L98H }(n=4) \\
\text { M220L } \\
\text { TR46/YI2IF/T289A }\end{array}$ \\
\hline Morio et $\mathrm{a}^{84}$ & $\begin{array}{l}\text { France } \\
\text { Single center (Nantes) }\end{array}$ & 50 & $\begin{array}{l}\text { EUCAST } \\
\text { CYP5IA sequencing }\end{array}$ & $\begin{array}{l}9 / 58 \text { isolates } \\
\text { in } 4 / 50(8 \%) \text { patients }\end{array}$ & $\begin{array}{l}\text { TR34/L98H }(n=5) \\
\text { M220T }(n=4) \\
\text { G54R }\end{array}$ \\
\hline Mortensen et al ${ }^{17}$ & $\begin{array}{l}\text { Denmark } \\
\text { Single center } \\
\text { (Copenhagen) }\end{array}$ & 133 & $\begin{array}{l}\text { EUCAST (itraconazole } \\
\text { screening agar) } \\
\text { CYP5IA sequencing }\end{array}$ & $6 / 133(4.5 \%)$ patients & $\begin{array}{l}\text { TR34/L98H }(n=2) \\
\text { M220I } \\
\text { M220K } \\
\text { Y43IC }\end{array}$ \\
\hline Terpstra et al ${ }^{85}$ & $\begin{array}{l}\text { The Netherlands } \\
\text { Multicenter }\end{array}$ & 653 & CYP5IA PCR & $59 / 1,072(5.5 \%)$ isolates & TR34/L98H predominantly \\
\hline
\end{tabular}

Note: ${ }^{a}$ Four of eleven patients had isolates with itraconazole MICs of $2 \mathrm{mg} / \mathrm{L}$, indicating intermediate susceptibility to itraconazole.

Abbreviations: CLSI, Clinical and Laboratory Standards Institute; N/A, not available; EUCAST, European Committee on Antimicrobial Susceptibility Testing; PCR, polymerase chain reaction, MICs, minimum inhibitory concentrations. 
A. fumigatus isolates. Mortensen et al found that all six patients with azole-resistant $A$. fumigatus isolates had been previously treated with azoles; ${ }^{.77}$ the authors reported a trend toward patients with the TR34/L98H phenotype having less azole exposure than the patients with M220 substitutions, suggesting a role for therapeutic azole exposure in the emergence of azole-resistant isolates in these latter patients. ${ }^{77}$ Burgel et al reported that azole-resistant $A$. fumigatus isolates were found in five of 25 (20\%) subjects with prolonged exposure to itraconazole within the previous 3 years, whereas a single azole-resistant (TR34/L98H) isolate was found in 84 patients without previous therapeutic azole exposure. ${ }^{14}$ Altogether these data strongly suggest that although azole-resistant isolates could be acquired through exposure to environmental strains (eg, TR34/L98H or more recently identified TR46/ $\mathrm{Y} 121 \mathrm{~F} / \mathrm{T} 289 \mathrm{~A}),{ }^{78}$ prolonged treatment with azoles is associated with in vivo selection of $A$. fumigatus strains exhibiting CYP51A mutations and azole resistance. Although most data on A. fumigatus resistance were obtained for itraconazole, an important aspect is the emergence of cross-resistance among $A$. fumigatus isolates. For example, A. fumigatus isolates resistant to itraconazole have been reported to be often resistant to posaconazole ${ }^{14}$ and/or voriconazole. ${ }^{79}$ Further, a recent study suggests that in vitro exposure of $A$. fumigatus isolates to voriconazole induces resistance to amphotericin $\mathrm{B} .{ }^{80}$ The emergence of azole-resistant $A$. fumigatus isolates could become a major concern in patients with $\mathrm{CF}$ and severe respiratory disease. Invasive aspergillosis is a frequent complication in CF patients undergoing lung transplantation, and Luong et al have recently shown that the risk of invasive aspergillosis after lung transplantation is increased when the explanted lung shows a positive intraoperative $A$. fumigatus culture. ${ }^{81}$ Because azole-resistant $A$. fumigatus isolates are associated with very high mortality (up to $88 \%$ ) in patients with invasive aspergillosis, ${ }^{74}$ we speculate that the selection of azole-resistant A. fumigatus isolates in the pretransplantation period may lead to untreatable invasive aspergillosis in the posttransplantation period.

\section{Conclusion and emerging perspectives}

A. fumigatus colonization and/or $A$. fumigatus sensitization with or without significant ABPA is found in a great proportion of $\mathrm{CF}$ patients. Although there is no doubt that ABPA is associated with airway damage and lung function decline, the effects of $A$. fumigatus colonization on lung function in the absence of ABPA are less well established. Some retrospective clinical studies found associations of
A. fumigatus colonization with CT scan abnormalities, greater risk of $\mathrm{CF}$ exacerbations/hospitalizations, and/or $\mathrm{FEV}_{1}$ decline, but these findings were not replicated in all studies. The availability of a growing number of oral antifungal triazole drugs together with the results of nonrandomized case series describing some positive effects of azole therapies makes it tempting to treat $\mathrm{CF}$ patients with these antifungal drugs. However, the only randomized controlled trial that has used itraconazole in CF patients showed no significant benefit. ${ }^{57}$ At this time, the best criteria for selecting patients who may benefit from azole therapy and the efficacy of this therapeutic approach have yet to be established. A recent study has suggested a novel immunologic classification of A. fumigatus in CF patients, ${ }^{13}$ but confirmation of the usefulness of this classification will require further studies. Because triazoles may have significant adverse effects and drug interactions, and because their prolonged use has been associated with the emergence of azole-resistant $A$. fumigatus isolates, it remains unclear whether CF patients benefit or not from azole therapy (Table 3). Progress in the understanding

Table 3 Summary of pros and cons of azole therapy in CF patients

\begin{tabular}{ll}
\hline Pros & Cons \\
\hline ABPA and Aspergillus & No randomized controlled trials have \\
sensitization are associated & evaluated the effects of adding azole to \\
with significant decline in lung & systemic steroids in CF patients with \\
function in CF patients & ABPA and/or Aspergillus sensitization \\
Aspergillus fumigatus & The only randomized controlled study \\
colonization has been & evaluating the effects of itraconazole \\
associated with lung function & in CF patients with Aspergillus \\
decline and increased risk of & colonization showed no effect on \\
exacerbations in some & exacerbation rate or FEV
\end{tabular}

(but not all) studies

Case reports and cases series have suggested beneficial role of azoles in these patients Azoles are easy to prescribe and to take because they are oral drugs

Azoles are usually well tolerated

Azoles may have the ability to eradicate $A$. fumigatus in $\mathrm{CF}$ patients

Therapeutic drug monitoring shows very variable azole concentrations in serum due to poor absorption, drug interactions, and/or poor adherence ${ }^{57}$ Therapeutic drug monitoring appears mandatory when prescribing azoles Voriconazole exposure is associated with photosensitization and skin cancer

Interaction of azoles with inhaled corticosteroids often results in adrenal insufficiency related to increase in serum steroid concentrations

Unproven

Prolonged exposure to azoles may results in azole resistance in A. fumigatus isolates

Abbreviations: CF, cystic fibrosis; ABPA, allergic bronchopulmonary aspergillosis; FEV , forced expiratory volume in I second. 
of the role of azoles in $\mathrm{CF}$ patients will require carefully designed randomized controlled clinical trials.

\section{Disclosure}

The authors report no conflicts of interest in relation to this work.

\section{References}

1. Davis PB. Cystic fibrosis since 1938. Am J Respir Crit Care Med. 2006; 173(5):475-482.

2. McCormick J, Mehta G, Olesen HV, et al. Comparative demographics of the European cystic fibrosis population: a cross-sectional database analysis. Lancet. 2010;375(9719):1007-1013.

3. Burgel PR, Bellis G, Olesen HV, et al. Future trends in cystic fibrosis demography in 34 European countries. Eur Respir J. 2015;46(1):133-141.

4. Cohen TS, Prince A. Cystic fibrosis: a mucosal immunodeficiency syndrome. Nat Med. 2012;18(4):509-519.

5. Ratner D, Mueller C. Immune responses in cystic fibrosis: are they intrinsically defective? Am J Respir Cell Mol Biol. 2012;46(6):715-722.

6. Chmiel JF, Aksamit TR, Chotirmall SH, et al. Antibiotic management of lung infections in cystic fibrosis. I. The microbiome, methicillin-resistant Staphylococcus aureus, gram-negative bacteria, and multiple infections. Ann Am Thorac Soc. 2014;11:1120-1129.

7. Chmiel JF, Aksamit TR, Chotirmall SH, et al. Antibiotic management of lung infections in cystic fibrosis. II. Nontuberculous mycobacteria, anaerobic bacteria, and fungi. Ann Am Thorac Soc. 2014;11(7): 1298-1306.

8. Paugam A, Baixench MT, Demazes-Dufeu N, et al. Characteristics and consequences of airway colonization by filamentous fungi in 201 adult patients with cystic fibrosis in France. Med Mycol. 2010;48 Suppl 1:S32-S36.

9. Fischer J, van Koningsbruggen-Rietschel S, Rietschel E, et al. Prevalence and molecular characterization of azole resistance in Aspergillus spp. isolates from German cystic fibrosis patients. J Antimicrob Chemother. 2014;69(6):1533-1536.

10. Sabino R, Ferreira JA, Moss RB, et al. Molecular epidemiology of Aspergillus collected from cystic fibrosis patients. J Cyst Fibros. 2015; 14(4):474-481.

11. Cystic Fibrosis Canada. The Canadian Cystic Fibrosis Registry. 2011 Annual Report. 2013. Available from: www.cysticfibrosis.ca/CanadianCF-Registry-2013-FINAL.pdf. Accessed September 8, 2016.

12. de Vrankrijker AM, van der Ent CK, van Berkhout FT, et al. Aspergillus fumigatus colonization in cystic fibrosis: implications for lung function? Clin Microbiol Infect. 2011;17(9):1381-1386.

13. Baxter CG, Dunn G, Jones AM, et al. Novel immunologic classification of aspergillosis in adult cystic fibrosis. J Allergy Clin Immunol. 2013;132(3):560-566.e10.

14. Burgel PR, Baixench MT, Amsellem M, et al. High prevalence of azole-resistant Aspergillus fumigatus in adults with cystic fibrosis exposed to itraconazole. Antimicrob Agents Chemother. 2012;56(2): 869-874.

15. Rocchi S, Richaud-Thiriez B, Barrera C, et al. Evaluation of mold exposure in cystic fibrosis patients' dwellings and allergic bronchopulmonary risk. J Cyst Fibros. 2015;14(2):242-247.

16. Delhaes L, Monchy S, Frealle E, et al. The airway microbiota in cystic fibrosis: a complex fungal and bacterial community--implications for therapeutic management. PLoS One. 2012;7(4):e36313.

17. Briard B, Bomme P, Lechner BE, et al. Pseudomonas aeruginosa manipulates redox and iron homeostasis of its microbiota partner Aspergillus fumigatus via phenazines. Sci Rep. 2015;5:8220.

18. Noni M, Katelari A, Dimopoulos G, et al. Inhaled corticosteroids and Aspergillus fumigatus isolation in cystic fibrosis. Med Mycol. 2014;52(7):715-722.

19. Burns JL, Emerson J, Stapp JR, et al. Microbiology of sputum from patients at cystic fibrosis centers in the United States. Clin Infect Dis. 1998; 27(1):158-163.
20. Baxter CG, Rautemaa R, Jones AM, et al. Intravenous antibiotics reduce the presence of Aspergillus in adult cystic fibrosis sputum. Thorax. 2013; 68(7):652-657.

21. Jubin V, Ranque S, Stremler Le Bel N, Sarles J, Dubus JC. Risk factors for Aspergillus colonization and allergic bronchopulmonary aspergillosis in children with cystic fibrosis. Pediatr Pulmonol. 2010;45(8):764-771.

22. de Valk HA, Klaassen CH, Yntema JB, et al. Molecular typing and colonization patterns of Aspergillus fumigatus in patients with cystic fibrosis. J Cyst Fibros. 2009;8(2):110-114.

23. Margalit A, Kavanagh K. The innate immune response to Aspergillus fumigatus at the alveolar surface. FEMS Microbiol Rev. 2015;39(5):670-687.

24. Eickmeier O, Rieber N, Eckrich J, Hector A, Graeppler-Mainka U, Hartl D. Immune response, diagnosis and treatment of allergic bronchopulmonary aspergillosis in cystic fibrosis lung disease. Curr Pharm Des. 2013;19(20):3669-3678.

25. Bhargava V, Tomashefski JF Jr, Stern RC, Abramowsky CR. The pathology of fungal infection and colonization in patients with cystic fibrosis. Hum Pathol. 1989;20(10):977-986.

26. Chaudhary N, Datta K, Askin FB, Staab JF, Marr KA. Cystic fibrosis transmembrane conductance regulator regulates epithelial cell response to Aspergillus and resultant pulmonary inflammation. Am J Respir Crit Care Med. 2012;185(3):301-310.

27. Mueller C, Braag SA, Keeler A, Hodges C, Drumm M, Flotte TR. Lack of cystic fibrosis transmembrane conductance regulator in CD3+ lymphocytes leads to aberrant cytokine secretion and hyperinflammatory adaptive immune responses. Am J Respir Cell Mol Biol. 2011;44(6):922-929.

28. Hamon Y, Jaillon S, Person C, et al. Proteolytic cleavage of the long pentraxin PTX3 in the airways of cystic fibrosis patients. Innate Immun. 2013; 19(6):611-622.

29. Heltshe SL, Mayer-Hamblett N, Burns JL, et al. Pseudomonas aeruginosa in cystic fibrosis patients with G551D-CFTR treated with ivacaftor. Clin Infect Dis. 2015;60(5):703-712.

30. Borman AM, Palmer MD, Delhaes L, et al. Lack of standardization in the procedures for mycological examination of sputum samples from $\mathrm{CF}$ patients: a possible cause for variations in the prevalence of filamentous fungi. Med Mycol. 2010;48 Suppl 1:S88-S97.

31. Masoud-Landgraf L, Badura A, Eber E, Feierl G, Marth E, Buzina W. Modified culture method detects a high diversity of fungal species in cystic fibrosis patients. Med Mycol. 2014;52(2):179-186.

32. Baxter CG, Jones AM, Webb K, Denning DW. Homogenisation of cystic fibrosis sputum by sonication--an essential step for Aspergillus PCR. $J$ Microbiol Methods. 2011;85(1):75-81.

33. Coughlan CA, Chotirmall SH, Renwick J, et al. The effect of Aspergillus fumigatus infection on vitamin $D$ receptor expression in cystic fibrosis. Am J Respir Crit Care Med. 2012;186(10):999-1007.

34. Nguyen LD, Viscogliosi E, Delhaes L. The lung mycobiome: an emerging field of the human respiratory microbiome. Front Microbiol. 2015;6:89.

35. Barnes RA. Early diagnosis of fungal infection in immunocompromised patients. JAntimicrob Chemother. 2008;61 Suppl 1:i3-i6.

36. Chotirmall SH, McElvaney NG. Fungi in the cystic fibrosis lung: bystanders or pathogens? Int J Biochem Cell Biol. 2014;52:161-173.

37. Massam J, Bitnun A, Solomon M, et al. Invasive aspergillosis in cystic fibrosis: a fatal case in an adolescent and review of the literature. Pediatr Infect Dis J. 2011;30(2):178-180.

38. Warren TA, Yau Y, Ratjen F, Tullis E, Waters V. Serum galactomannan in cystic fibrosis patients colonized with Aspergillus species. Med Mycol. 2012;50(6):658-660.

39. van Toorenenbergen AW. Between-laboratory quality control of automated analysis of IgG antibodies against Aspergillus fumigatus. Diagn Microbiol Infect Dis. 2012;74(3):278-281.

40. Baxter CG, Denning DW, Jones AM, Todd A, Moore CB, Richardson MD. Performance of two Aspergillus IgG EIA assays compared with the precipitin test in chronic and allergic aspergillosis. Clin Microbiol Infect. 2013;19(4):E197-E204.

41. Oliva A, Flori P, Hennequin C, et al. Evaluation of the Aspergillus Western blot IgG kit for diagnosis of chronic aspergillosis. J Clin Microbiol. 2015;53:248-254. 
42. Singh B, Singh S, Asif AR, Oellerich M, Sharma GL. Allergic aspergillosis and the antigens of Aspergillus fumigatus. Curr Protein Pept Sci. 2014; 15(5):403-423.

43. Hilliard T, Edwards S, Buchdahl R, et al. Voriconazole therapy in children with cystic fibrosis. J Cyst Fibros. 2005;4(4):215-220.

44. Maturu VN, Agarwal R. Prevalence of Aspergillus sensitization and allergic bronchopulmonary aspergillosis in cystic fibrosis: systematic review and meta-analysis. Clin Exp Allergy. 2015;45(12):1765-1778.

45. Wojnarowski C, Eichler I, Gartner C, et al. Sensitization to Aspergillus fumigatus and lung function in children with cystic fibrosis. Am J Respir Crit Care Med. 1997;155(6):1902-1907.

46. Kraemer R, Delosea N, Ballinari P, Gallati S, Crameri R. Effect of allergic bronchopulmonary aspergillosis on lung function in children with cystic fibrosis. Am J Respir Crit Care Med. 2006;174(1):1211-1220.

47. Fillaux J, Bremont F, Murris M, et al. Assessment of Aspergillus sensitization or persistent carriage as a factor in lung function impairment in cystic fibrosis patients. Scand J Infect Dis. 2012;44(11):842-847.

48. Peetermans M, Goeminne P, De Boeck C, Dupont LJ. IgE sensitization to Aspergillus fumigatus is not a bystander phenomenon in cystic fibrosis lung disease. Chest. 2014;146(3):e99-e100.

49. Baxter CG, Moore CB, Jones AM, Webb AK, Denning DW. IgEmediated immune responses and airway detection of Aspergillus and Candida in adult cystic fibrosis. Chest. 2013;143(5):1351-1357.

50. Amin R, Dupuis A, Aaron SD, Ratjen F. The effect of chronic infection with Aspergillus fumigatus on lung function and hospitalization in patients with cystic fibrosis. Chest. 2010;137(1):171-176.

51. Ramsey KA, Ranganathan S, Park J, et al. Early respiratory infection is associated with reduced spirometry in children with cystic fibrosis. Am J Respir Crit Care Med. 2014;190(10):1111-1116.

52. de Boer K, Vandemheen KL, Tullis E, et al. Exacerbation frequency and clinical outcomes in adult patients with cystic fibrosis. Thorax. 2011; 66(8):680-685.

53. Reihill JA, Moore JE, Elborn JS, Ennis M. Effect of Aspergillus fumigatus and Candida albicans on pro-inflammatory response in cystic fibrosis epithelium. J Cyst Fibros. 2011;10(6):401-406.

54. McMahon MA, Chotirmall SH, McCullagh B, Branagan P, McElvaney NG, Logan PM. Radiological abnormalities associated with Aspergillus colonization in a cystic fibrosis population. Eur J Radiol. 2012;81(3):e197-e202.

55. Elphick HE, Southern KW. Antifungal therapies for allergic bronchopulmonary aspergillosis in people with cystic fibrosis. Cochrane Database Syst Rev. 2012;(6):CD002204.

56. Shoseyov D, Brownlee KG, Conway SP, Kerem E. Aspergillus bronchitis in cystic fibrosis. Chest. 2006;130(1):222-226.

57. Aaron SD, Vandemheen KL, Freitag A, et al. Treatment of Aspergillus fumigatus in patients with cystic fibrosis: a randomized, placebocontrolled pilot study. PLoS One. 2012;7(4):e36077.

58. Lestner J, Hope WW. Itraconazole: an update on pharmacology and clinical use for treatment of invasive and allergic fungal infections. Expert Opin Drug Metab Toxicol. 2013;9(7):911-926.

59. Thompson GR 3rd, Lewis JS 2nd. Pharmacology and clinical use of voriconazole. Expert Opin Drug Metab Toxicol. 2010;6(1):83-94.

60. Jacinto PL, Chandrasekar P. Safety of posaconazole. Expert Opin Drug Saf. 2013;12(12):265-274.

61. Billaud EM, Guillemain R, Berge M, et al. Pharmacological considerations for azole antifungal drug management in cystic fibrosis lung transplant patients. Med Mycol. 2010;48 Suppl 1:S52-SS9.

62. Shakeri-Nejad K, Stahlmann R. Drug interactions during therapy with three major groups of antimicrobial agents. Expert Opin Pharmacother. 2006;7(6):639-651.

63. Skov M, Main KM, Sillesen IB, Muller J, Koch C, Lanng S. Iatrogenic adrenal insufficiency as a side-effect of combined treatment of itraconazole and budesonide. Eur Respir J. 2002;20(1):127-133.

64. Albert BB, Jaksic M, Ramirez J, et al. An unusual cause of growth failure in cystic fibrosis: a salutary reminder of the interaction between glucocorticoids and cytochrome P450 inhibiting medication. J Cyst Fibros. 2015;14(4):e9-e11.
65. Gilchrist FJ, Cox KJ, Rowe R, et al. Itraconazole and inhaled fluticasone causing hypothalamic-pituitary-adrenal axis suppression in adults with cystic fibrosis. J Cyst Fibros. 2013;12(4):399-402.

66. Jones W, Chastain CA, Wright PW. Iatrogenic cushing syndrome secondary to a probable interaction between voriconazole and budesonide. Pharmacotherapy. 2014;34(7):e116-e119.

67. Pilmis B, Coignard-Biehler H, Jullien V, et al. Iatrogenic Cushing's syndrome induced by posaconazole. Antimicrob Agents Chemother. 2013; 57(11):5727-5728.

68. De Boeck K, Vermeulen F, Wanyama S, Thomas M. Inhaled corticosteroids and lower lung function decline in young children with cystic fibrosis. Eur Respir J. 2011;37(5):1091-1095.

69. Rondeau S, Couderc L, Dominique S, et al. High frequency of voriconazole-related phototoxicity in cystic fibrosis patients. Eur Respir J. 2012;39(3):782-784.

70. Epaulard O, Leccia MT, Blanche S, et al. Phototoxicity and photocarcinogenesis associated with voriconazole. Med Mal Infect. 2011; 41(12):639-645

71. Howard SJ, Arendrup MC. Acquired antifungal drug resistance in Aspergillus fumigatus: epidemiology and detection. Med Mycol. 2011;49 Suppl 1:S90-S95.

72. Howard SJ, Cerar D, Anderson MJ, et al. Frequency and evolution of Azole resistance in Aspergillus fumigatus associated with treatment failure. Emerg Infect Dis. 2009;15(7):1068-1076.

73. Seyedmousavi S, Mouton JW, Melchers WJ, Bruggemann RJ, Verweij PE. The role of azoles in the management of azole-resistant aspergillosis: from the bench to the bedside. Drug Resist Update. 2014;17(3):37-50.

74. van der Linden JW, Arendrup MC, Warris A, et al. Prospective multicenter international surveillance of azole resistance in Aspergillus fumigatus. Emerg Infect Dis. 2015;21(6):1041-1044.

75. Verweij PE, Snelders E, Kema GH, Mellado E, Melchers WJ. Azole resistance in Aspergillus fumigatus: a side-effect of environmental fungicide use? Lancet Infect Dis. 2009;9(12):789-795.

76. Klaassen CH, De Valk HA, Curfs-Breuker IM, Meis JF. Novel mixedformat real-time PCR assay to detect mutations conferring resistance triazoles in Aspergillus fumigatus and prevalence of multi-triazole resistance among clinical isolates in the Netherlands. $J$ Antimicrob Chemother. 2010;65(5):901-905.

77. Mortensen KL, Jensen RH, Johansen HK, et al. Aspergillus species and other moulds in respiratory samples from cystic fibrosis patients: a laboratory-based study with focus on azole resistance in Aspergillus fumigatus. J Clin Microbiol. 2011;49(6):2243-2251.

78. Lavergne RA, Morio F, Favennec L, et al. First description of azoleresistant Aspergillus fumigatus due to TR46/Y121F/T289A mutation in France. Antimicrob Agents Chemother. 2015;59(7):4331-4335.

79. Kidd SE, Goeman E, Meis JF, Slavin MA, Verweij PE. Multi-triazoleresistant Aspergillus fumigatus infections in Australia. Mycoses. 2015;58(6):350-355.

80. Rajendran R, Mowat E, Jones B, Williams C, Ramage G. Prior in vitro exposure to voriconazole confers resistance to amphotericin $\mathrm{B}$ in Aspergillus fumigatus biofilms. Int J Antimicrob Agents. 2015;46(3):342-345.

81. Luong ML, Chaparro C, Stephenson A, et al. Pretransplant Aspergillus colonization of cystic fibrosis patients and the incidence of post-lung transplant invasive aspergillosis. Transplantation. 2014;97(3):351-357.

82. Amorim A, Guedes-Vaz L, Araujo R. Susceptibility to five antifungals of Aspergillus fumigatus strains isolated from chronically colonised cystic fibrosis patients receiving azole therapy. Int J Antimicrob Agents. 2010;35(4):396-399.

83. Bader O, Weig M, Reichard U, et al. cyp51A-Based mechanisms of Aspergillus fumigatus azole drug resistance present in clinical samples from Germany. Antimicrob Agents Chemother. 2013;57(8):3513-3517.

84. Morio F, Aubin GG, Danner-Boucher I, et al. High prevalence of triazole resistance in Aspergillus fumigatus, especially mediated by TR/ L98H, in a French cohort of patients with cystic fibrosis. J Antimicrob Chemother. 2012;67(8):1870-1873.

85. Terpstra PD, van Westreenen MJ, Croughs P, et al. Filamentous fungi in the Netherlands among CF patients. Mycoses. 2011;54 Suppl 2:152. 


\section{Publish your work in this journal}

Infection and Drug Resistance is an international, peer-reviewed openaccess journal that focuses on the optimal treatment of infection (bacterial, fungal and viral) and the development and institution of preventive strategies to minimize the development and spread of resistance. The journal is specifically concerned with the epidemiology of antibiotic resistance and the mechanisms of resistance development and diffusion in both hospitals and the community. The manuscript management system is completely online and includes a very quick and fair peerreview system, which is all easy to use. Visit http://www.dovepress.com/ testimonials.php to read real quotes from published authors.

Submit your manuscript here: https://www.dovepress.com/infection-and-drug-resistance-journal 